\title{
Aspects of classical and quantum motion on a flux cone
}

\author{
E. S. Moreira, Jr.* \\ Instituto de Física Teórica, Universidade Estadual Paulista, Rua Pamplona 145, 01405-900-São Paulo, São Paulo, Brazil
}

(Received 7 January 1998)

\begin{abstract}
Motion of a nonrelativistic particle on a cone with a magnetic flux running through the cone axis (a "flux cone") is studied. It is expressed as the motion of a particle moving on the Euclidean plane under the action of a velocity-dependent force. The probability fluid ("quantum flow") associated with a particular stationary state is studied close to the singularity, demonstrating nontrivial Aharonov-Bohm effects. For example, it is shown that, near the singularity, quantum flow departs from classical flow. In the context of the hydrodynamical approach to quantum mechanics, quantum potential due to the conical singularity is determined, and the way it affects quantum flow is analyzed. It is shown that the winding number of classical orbits plays a role in the description of the quantum flow. The connectivity of the configuration space is also discussed. [S1050-2947(98)03508-2]

PACS number(s): 03.65.Bz, 04.62.+v
\end{abstract}

\section{INTRODUCTION}

Usually, when the quantum description of a system is nontrivial, so is the classical one. However, this is not always the case. There is a number of examples whose intrinsic quantum effects do not have a classical analog. Notable among these is the Aharonov-Bohm (AB) effect [1]. In this setup, the magnetic field vanishes everywhere except inside a thin flux tube. As there is no Lorentz force, classically particles are free, and are not affected by the background. However, in the quantum scattering problem the background leads to a nontrivial scattering, which is confirmed by experiment [2].

The $\mathrm{AB}$ effect is a consequence of the interaction of quantum matter with a nearly trivial affine connection, and it is present in every gauge theory, including gravity. The analog of the $\mathrm{AB}$ setup in gravitation is a conical background [3-5]. The geometry is flat everywhere apart from a symmetry axis. As in the case of a thin flux tube, the problem of studying quantum mechanics on cones amounts to solving the usual equations in flat space with nontrivial boundary conditions. Solutions of these equations lead to geometrical AharonovBohm effects [6-9].

Quantum theory in conical backgrounds has been studied over two decades [10], and investigations intensified in the mid 1980s [11] very much due to the importance in cosmology of cosmic strings. More recently, interest in the subject was renewed in the context of quantum mechanics of black holes, where one encounters conical singularities (see, e.g., Ref. [12], and references therein). Besides these and other practical motivations to study quantum theory in conical backgrounds, there is another more academic one [6-9]. That is, the study of quantum mechanics in a nearly trivial gravitational background may shed some light on the profound problems of combining quantum mechanics and general relativity.

In this work classical and quantum effects caused by a conical singularity on the motion of a particle are studied. On

*Electronic address: moreira@axp.ift.unesp.br various occasions the $\mathrm{AB}$ setup is coupled to a conical geometry (flux cone), so that a comparison of the corresponding $\mathrm{AB}$ effects is possible. The paper is organized as follows. An account on the conical geometry and classical motion on a cone is given in Sec. II (this section comprises a review of early works by Deser, Gerbert, Jackiw, and 't Hooft [13,6$8]$ ), commenting on points which have been overlooked in the literature. In Sec. III, classical motion on a flux cone is studied. Quantization is implemented in Sec. IV, where the issue of boundary conditions at the singularity is considered, and a particular one is chosen, motivated by regularization arguments $[14,15]$. Corresponding stationary states are obtained and used to build up a state to probe the singularity. This leads, in Sec. V, to a study of quantum flow, showing nontrivial effects due to the conical singularity. Such effects are caused by a nonvanishing quantum potential whose features are mentioned. Connectivity of the configuration space is discussed by taking into account the behavior of quantum flow at the singularity. Sec. VI is a summary, including possible extensions of this study. An account on the hydrodynamical approach to quantum mechanics (whose elements are used in Sec. V) is given in the Appendix.

\section{CONE}

As mentioned previously this section is essentially a review of material in Refs. [13,6-8]. A cone is a twodimensional space with a $\delta$-function curvature singularity [16]. The line element may be written as

$$
\begin{aligned}
d l^{2} & =g_{i j} d x^{i} d x^{j} \\
& =\left[\delta_{i j}+\left(\alpha^{-2}-1\right) \frac{x^{i} x^{j}}{r^{2}}\right] d x^{i} d x^{j},
\end{aligned}
$$

where the coordinate $x^{i}(i=1,2)$ runs from $-\infty$ to $\infty, r^{2}$ $:=\delta_{i j} x^{i} x^{j}$ and $\alpha$ is a positive parameter [13]. Imagining the conical surface embedded in a three-dimensional Euclidean space, $\left\{x^{1}, x^{2}\right\}$ are Cartesian coordinates on a plane perpendicular to the symmetry axis of the cone (Fig. 1). From Eq. 


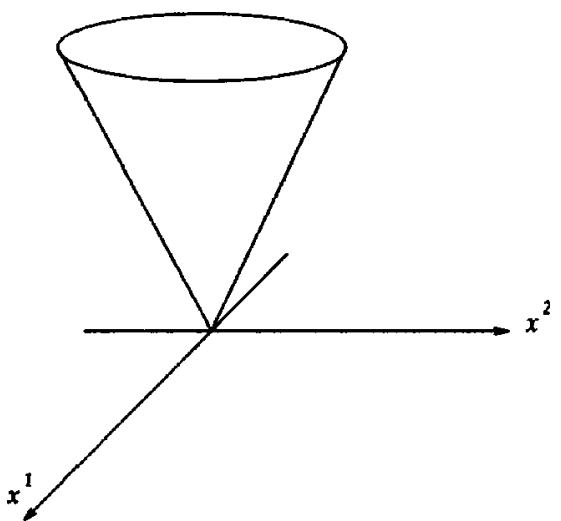

FIG. 1. Cone embedded in a three-dimensional Euclidean space.

(1), one sees that the conical singularity is located at the origin, and that, when $\alpha=1$, the cone becomes the Euclidean plane.

Using polar coordinates $\left(x^{1}=r \cos \theta, x^{2}=r \sin \theta\right)$, Eq. (1) can be rewritten as $d l^{2}=\alpha^{-2} d r^{2}+r^{2} d \theta^{2}$, where the conical singularity is now hidden by the coordinate singularity at the origin. Note that the coordinates $(r, \theta)$ and $(r, \theta+2 \pi)$ label the same point, $(r, \theta) \sim(r, \theta+2 \pi)$. A further simplification of the line element can be made by rescaling $\{r, \theta\}$ as

$$
\rho=\alpha^{-1} r, \quad \varphi=\alpha \theta
$$

resulting in $d l^{2}=d \rho^{2}+\rho^{2} d \varphi^{2}$, showing the flatness of the cone. However, as a consequence of the rescaling,

$$
(\rho, \varphi) \sim(\rho, \varphi+2 \pi \alpha)
$$

Coordinates $\{\rho, \varphi\}$ are polar coodinates on the surface of the cone. The angle of the missing wedge (extra wedge, when $\alpha>1$ ) is the deficit angle of the cone $\mathcal{D}=2 \pi(1-\alpha)$ (Fig. 2).

At this point it should be noted that the Euclidean plane and a cone have the same topology, differing in their geometry-the former is globally flat whereas the latter is not. To see this, one smooths the cone by replacing its tip by a tangential spherical cap of radius $a$ (Fig. 3). Clearly the resulting surface is simply connected, a fact that does not change when $a \rightarrow 0$ and the idealized cone is recovered. Though the cone is a simply connected background, the con-

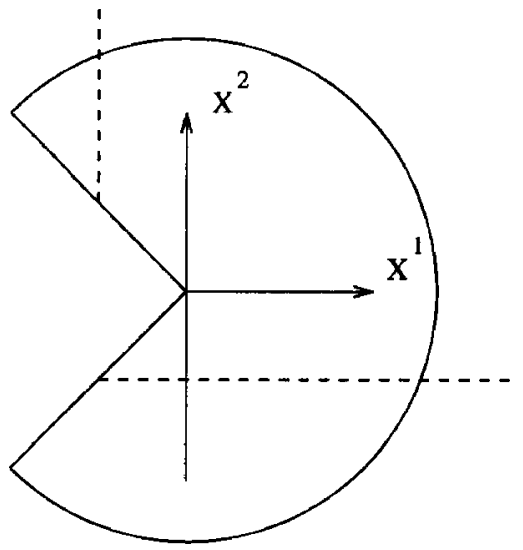

FIG. 2. Singular Cartesian frame.

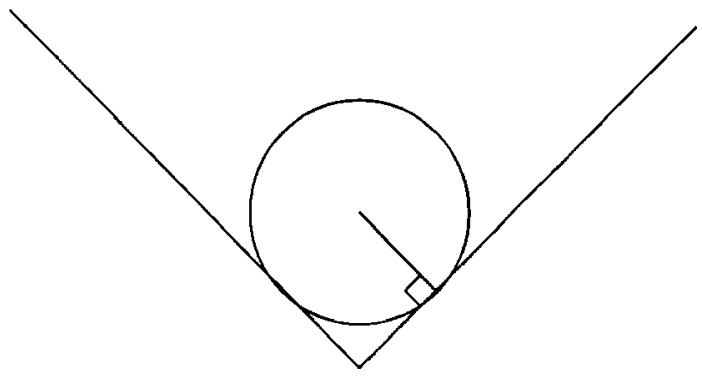

FIG. 3. Smoothing the cone.

figuration space of a quantum particle living on it may be nonsimply connected (see Sec. V).

It follows from Eq. (3) that the "Cartesian" coordinates defined by $X^{1}:=\rho \cos \varphi$ and $X^{2}:=\rho \sin \varphi$ (Fig. 2) are singular if $\mathcal{D} \neq 0$ : in terms of $\left\{X^{1}, X^{2}\right\}$ the metric tensor is Euclidean everywhere except on the rays defining the borders of the wedge, where these coordinates are discontinuous functions of $\varphi$. (Clearly the borders of the wedge can be arbitrarily rotated by redefining the interval of length $2 \pi \alpha$ over which $\varphi$ ranges.) Accordingly, the Levi-Civita connection vanishes everywhere except on the borders of the wedge, where it is singular. The nonvanishing connection "tells" the rest of the space that there is a curvature singularity at the origin, and this nontrivial behavior is the very one responsible for the geometrical Aharonov-Bohm-like effects that will be seen in this work.

Classical motion of a free particle with mass $M$ on a conical surface may be determined from the Lagrangian,

$$
\begin{aligned}
\mathcal{L} & =\frac{1}{2} M(d l / d t)^{2} \\
& =\alpha^{-2}\left[\frac{1}{2} M \dot{\mathbf{x}}^{2}-\left(1-\alpha^{2}\right) \frac{\ell^{2}}{2 M r^{2}}\right] .
\end{aligned}
$$

The line element $d l$ is given by Eq. (1), the dot denotes differentiation with respect to the time $t, \mathbf{x}:=\left(x^{1}, x^{2}\right)$, and $\ell:=\mathbf{x} \times M \dot{\mathbf{x}}$ is the kinematical angular momentum (note that $\left.\mathbf{a} \times \mathbf{b}:=\epsilon_{i j} a^{i} b^{j}\right)$. The Euler-Lagrange equations of motion, i.e., the geodesic equations on the cone, follow from Eq. (4),

$$
M \ddot{\mathbf{x}}=-\left(1-\alpha^{2}\right) \frac{\ell^{2}}{M r^{3}} \mathbf{e}_{r},
$$

where $\mathbf{e}_{r}:=\mathbf{x} / r$. One can regard Eq. (5) as the equation of motion of a particle moving on the Euclidean plane under the action of an angular-momentum-dependent central force, which is attractive for $\alpha<1$ and repulsive for $\alpha>1$ [17]. Clearly when $\ell=0$ the motion is radial and uniform.

The geometrical force in Eq. (5) ranks somewhere between an inertial force and a Newtonian force [5,18]. Indeed by changing from $\left\{x^{1}, x^{2}\right\}$ to $\left\{X^{1}, X^{2}\right\}$, the force is "gauged away" everywhere apart from the borders of the wedge. Therefore, unless $\alpha=1$, trajectories crossing these rays are broken straight lines, with uniform motion (dashed line in Fig. 2). Integration of Eq. (5) $[7,18]$ shows that particles wind

$$
w=[1 / 2 \alpha]
$$




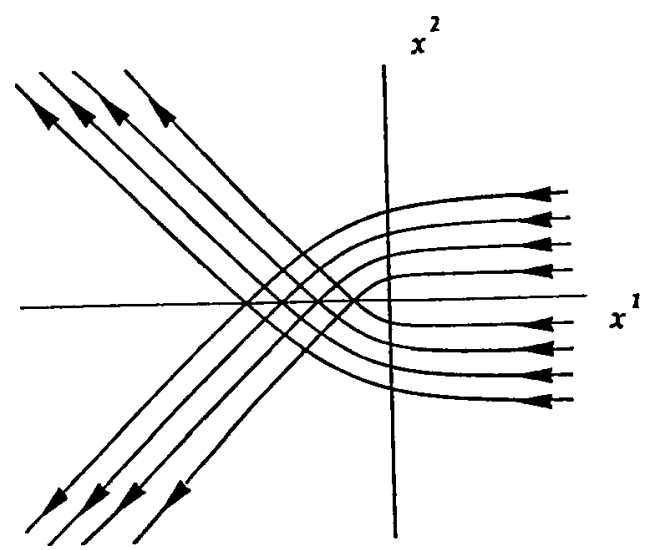

FIG. 4. Classical motion on a cone.

([x] denotes the integral part of $x$ ) times around the conical singularity (Fig. 4).

\section{FLUX CONE}

A cone may be immersed in a magnetic field pointing along its axis, and being homogeneous in that direction, by choosing a vector potential $\mathbf{A}(\mathbf{x}, t)$ with a vanishing component in that direction. The Lagrangian of a particle with charge $e$ moving in this background is given by

$$
\mathcal{L}^{\prime}=\mathcal{L}+\frac{e}{c} \dot{\mathbf{x}} \cdot \mathbf{A} .
$$

Thus the canonical momentum associated with $\mathbf{x}$ is

$$
\begin{aligned}
\mathbf{p} & =\alpha^{-2}\left[M \dot{\mathbf{x}}+\left(\alpha^{2}-1\right) \frac{\ell}{r} \mathbf{e}_{\theta}\right]+\frac{e}{c} \mathbf{A} \\
& =M \dot{\mathbf{x}}+\left(\alpha^{-2}-1\right) M \dot{r} \mathbf{e}_{r}+\frac{e}{c} \mathbf{A} .
\end{aligned}
$$

It follows from Eq. (8) that

$$
\mathbf{x} \times \mathbf{p}=\ell+\frac{e}{c} \mathbf{x} \times \mathbf{A},
$$

which is the usual expression for the canonical angular momentum on the Euclidean plane. The Hamiltonian is given by

$$
\begin{aligned}
\mathcal{H} & =\mathbf{p} \cdot \dot{\mathbf{x}}-\mathcal{L}^{\prime} \\
& =\frac{\alpha^{2}}{2 M}\left(\mathbf{p}-\frac{e}{c} \mathbf{A}\right)^{2}+\frac{\left(1-\alpha^{2}\right)}{2 M r^{2}}\left[\mathbf{x} \times\left(\mathbf{p}-\frac{e}{c} \mathbf{A}\right)\right]^{2} .
\end{aligned}
$$

A magnetic flux $\Phi(t)$ running through the axis of the cone can be introduced by choosing

$$
\mathbf{A}=\frac{\Phi}{2 \pi r} \mathbf{e}_{\theta},
$$

where $\mathbf{e}_{\theta}:=(-\sin \theta, \cos \theta)$. Now there is a new singularity at the origin which is a $\delta$ function magnetic field. Due to the cylindrical symmetry, the canonical angular momentum as given by Eq. (9) is conserved,

$$
\frac{d}{d t}\left(\ell+\frac{e \Phi}{2 \pi c}\right)=0 .
$$

The equations of motion following from Eq. (7) are given by Eq. (5), with the induced electric force $-e\left(\partial_{t} \Phi\right) \mathbf{e}_{\theta} / 2 \pi c r$ added on the right-hand side. This electric force prevents the orbital angular momentum $\ell$ being a constant of motion, which is consistent with Eq. (12). Clearly the above expressions reduce to the familiar ones on the plane [19] when $\alpha$ $=1$.

The Aharonov-Bohm setup may be combined with the conical geometry by taking $\Phi$ constant, in which case the electric force vanishes, and Eq. (5) still holds. Obviously the same conclusion may be reached by realizing that the electromagnetic Lagrangian in Eq. (7) is a total derivative, $e \Phi \dot{\theta} / 2 \pi c$, and consequently does not affect classical motion. However, as is well known [1], this is not the case in quantum theory, which is the subject of the following sections. [In the following sections, $\mathbf{A}$ is given by Eq. (11) with constant $\Phi$.]

\section{HAMILTONIAN OPERATOR AND STATIONARY STATES}

The Hamiltonian operator $H$ can be obtained from Eq. (10) by the usual substitution [7], $\mathbf{p} \rightarrow-i \hbar \nabla$. The invariance of the Schrödinger equation under the gauge transformation $\mathbf{A}(\mathbf{x}) \rightarrow \mathbf{A}(\mathbf{x})+\boldsymbol{\nabla} \chi(\mathbf{x}), \quad \psi(\mathbf{x}, t) \rightarrow \exp \{i(e / \hbar c) \chi(\mathbf{x})\} \psi(\mathbf{x}, t) \quad$ al lows the choice $\chi(\mathbf{x})=(-\Phi / 2 \pi) \theta(\mathbf{x})$, thereby gauging away the vector potential everywhere, except on an arbitrary ray where the polar angle $\theta$ is a discontinuous function of $\mathbf{x}$ [20]. This singular gauge is analogous to the singular Cartesian coordinates $\left\{X^{1}, X^{2}\right\}$. It might have been anticipated that A cannot vanish everywhere around the origin since $\oint \mathbf{A} \cdot d \mathbf{x}=\Phi$. Similar reasoning may be applied to the LeviCivita connection of a conical geometry (see Ref. [21] and references therein).

Defining $\sigma:=-e \Phi / c h$, the transformed wave function is

$$
\psi^{\prime}(\mathbf{x}, t)=\exp \{i \sigma \theta\} \psi(\mathbf{x}, t) .
$$

It then follows from Eq. (10), (2), and (3) that

$$
\begin{gathered}
H=-\frac{\hbar^{2}}{2 M} \frac{1}{\rho} \frac{\partial}{\partial \rho}\left(\rho \frac{\partial}{\partial \rho}\right)+\frac{L^{2}}{2 M \rho^{2}}, \\
\psi(\rho, \varphi+2 \pi \alpha)=\exp \{i 2 \pi \sigma\} \psi(\rho, \varphi),
\end{gathered}
$$

where

$$
L:=-i \hbar \frac{\partial}{\partial \varphi}
$$

and the prime in $\psi^{\prime}$ has been dropped. As $H$ in Eq. (14) is just the free Hamiltonian operator on the plane written in polar coordinates [which is not surprising since $\left\{X^{1}, X^{2}\right\}$ is a (singular) Cartesian frame], the interaction with the magnetic flux and the conical geometry manifest themselves only through Eq. (15). In fact the twisted boundary condition (15) states that the wave function is not single valued for noninteger values of the flux parameter $\sigma$, and therefore is not 
continuous along some ray. This corresponds to (and is compatible with) the fact that $H$, as given by Eq. (14), disguises a singular vector potential which, as mentioned above, is not defined everywhere. Note also that, according to Eq. (15), $\psi$ must either vanish or diverge at the origin (for noninteger $\sigma)$, otherwise an inconsistency results when a loop is shrunk around $\rho=0$ [22].

Considering Eqs. (14) and (15), it follows from the Schrödinger equation

$$
\frac{d}{d t} \int_{0}^{\infty} d \rho \rho \int_{0}^{2 \pi \alpha} d \varphi \psi \psi^{*}=\lim _{\rho \rightarrow 0} \int_{0}^{2 \pi \alpha} d \varphi \rho \mathcal{J}_{\rho},
$$

where $\mathcal{J}_{\rho}$ is the usual expression for the radial component of the probability current on the plane,

$$
\mathcal{J}_{\rho}=\frac{1}{M} \operatorname{Re}\left[\psi^{*} \frac{\hbar}{i} \frac{\partial \psi}{\partial \rho}\right], \quad \mathcal{J}_{\varphi}=\frac{1}{M} \operatorname{Re}\left[\psi^{*} \frac{\hbar}{i \rho} \frac{\partial \psi}{\partial \varphi}\right] .
$$

In obtaining Eq. (17), it has been assumed that the wave function vanishes at infinity. The right-hand side of Eq. (17) is the net probability crossing an infinitesimally small circle around the singularity. Equating Eq. (17) to zero amounts to the statement that the singularity at the origin is neither a source nor a sink (probability is conserved), which is automatically guaranteed if

$$
\lim _{\rho \rightarrow 0} \int_{0}^{2 \pi \alpha} d \varphi \rho\left(\psi^{*} \frac{\partial \phi}{\partial \rho}-\frac{\partial \psi^{*}}{\partial \rho} \phi\right)=0 .
$$

Expression (19) is the condition for self-adjointness of the Hamiltonian operator (14), $\langle\psi|H| \phi\rangle=\langle\phi|H| \psi\rangle^{*}$.

If $R_{k, m}(\rho)$ are functions such that

$$
\left[\rho \frac{\partial}{\partial \rho}\left(\rho \frac{\partial}{\partial \rho}\right)-\left(\frac{m+\sigma}{\alpha}\right)^{2}+(k \rho)^{2}\right] R_{k, m}(\rho)=0,
$$

then

$$
\psi_{k, m}(\rho, \varphi)=\frac{1}{\sqrt{2 \pi \alpha}} R_{k, m}(\rho) e^{i(m+\sigma) \varphi / \alpha},
$$

where $0 \leqslant k<\infty$ and $m$ is an integer, are simultaneous eigenfunctions of $H$ and $L$ with eigenvalues $\hbar^{2} k^{2} / 2 M$ and ( $m$ $+\sigma) \hbar / \alpha$, respectively. Note that the effect of the magnetic flux and of the conical geometry on the eigenvalues of $L$ is to shift and to rescale them, respectively. For a particle with spin there is also a shift due to a coupling between the spin and the deficit angle $[8,23]$.

In order that the stationary states $\psi_{k, m}$ span a space of wave functions in which probability is conserved, they must satisfy Eq. (19),

$$
\lim _{\rho \rightarrow 0} \rho\left(R_{k^{\prime}, m}^{*} \frac{\partial R_{k, m}}{\partial \rho}-\frac{\partial R_{k^{\prime}, m}^{*}}{\partial \rho} R_{k, m}\right)=0,
$$

where the orthonormality relation $\int_{0}^{2 \pi \alpha} d \varphi \exp \{i \varphi(m-n) / \alpha\}$ $=2 \pi \alpha \delta_{m n}$ has been used. Expression (22) is the condition for self-adjointness of the square of the operator $P_{\rho}:=-i \hbar\left(\partial_{\rho}\right.$ $+1 / 2 \rho)$, and is itself self-adjoint if

$$
\lim _{\rho \rightarrow 0} \rho\left(\phi \psi^{*}\right)=0
$$

as may easily be shown by equating $\int_{0}^{\infty} d \rho \rho\left[\phi\left(P_{\rho} \psi\right)^{*}\right.$ $\left.-\psi^{*}\left(P_{\rho} \phi\right)\right]$ to zero. One sees from Eq. (23) that $R_{k, m}(\rho)$ may diverge mildly at the origin [which also applies to $\mathcal{J}_{\rho}$ in Eq. (17)], and still be compatible with conservation of probability. A mild divergence would not spoil the square integrability of the wave function, since a behavior $\psi \sim 1 / \rho^{\nu}$ with $\nu<1$ yields $\int_{0}^{\epsilon} d \rho \rho|\psi|^{2} \propto \epsilon^{2(1-\nu)}$, which vanishes with $\epsilon$. Note that in order to satisfy Eq. (23) one must have $\nu<\frac{1}{2}$. Obviously a logarithmic divergence also passes this test, since it is weaker than the $1 / \rho^{\nu}$ divergence.

In the following only wave functions which are finite at the singularity will be considered. In quantum mechanics on the flux cone, finiteness is motivated by the fact that it arises naturally when the singularities are smoothed by some regularization procedure [14,15]. (Divergent wave functions were considered in Refs. $[14,15]$.) Therefore, one takes as solutions of Eq. (20) Bessel functions of the first kind,

$$
R_{k, m}(\rho)=J_{|m+\sigma| / \alpha}(k \rho),
$$

which being finite at the origin satisfy Eq. (23) and consequently Eq. (22). As a check we may verify that Eq. (24) indeed satisfies Eq. (22) by observing that

$$
\frac{d J_{\nu}(x)}{d x}=\frac{1}{2}\left[J_{\nu-1}(x)-J_{\nu+1}(x)\right] .
$$

Hence the complete set of stationary states $\psi_{k, m}$ is given by Eqs. (21) and (24).

A particular state to probe the singularity can be found as follows. The general expression for a stationary state of energy $\hbar^{2} k^{2} / 2 M$ is given by

$$
\psi_{k}(\rho, \varphi)=\sum_{m=-\infty}^{\infty} c_{m} \psi_{k, m}(\rho, \varphi)
$$

The coefficients $c_{m}$ are determined by considering the Fourier expansion of a plane wave

$$
\exp \{-i a \cos \phi\}=\sum_{m=-\infty}^{\infty}(-i)^{|m|} J_{|m|}(a) e^{i m \phi},
$$

and letting the magnetic flux and the conical geometry act on it. This involves shifting and rescaling the angular momentum of the modes, as mentioned previously, leading to

$$
c_{m}^{(\delta, \alpha)}:=\sqrt{2 \pi \alpha} \exp \left\{-\frac{i \pi}{2 \alpha}|m-\delta|\right\},
$$

where the flux parameter has been redefined to be $\delta:=-\sigma$ in order to compare the result with earlier work. The stationary state (26) with $c_{m}=c_{m}^{(\delta, \alpha)}$ will be denoted $\psi_{k}^{(\delta, \alpha)}$. Clearly,

$$
\begin{aligned}
\psi_{k}^{(0,1)}(\rho, \varphi) & =\exp \{-i k \rho \cos \varphi\} \\
& \equiv \exp \left\{-i k X^{1}\right\}
\end{aligned}
$$


(Note that when $\alpha=1$, both $\left\{x^{1}, x^{2}\right\}$ and $\left\{X^{1}, X^{2}\right\}$ are genuine Cartesian coordinates which coincide.) The stationary states $\psi^{(\delta, 1)}$ and $\psi^{(0, \alpha)}$ have previously been considered in the literature (in Refs. [1] and [9,7], respectively) in the context of scattering. Section V considers the probability fluid (see the Appendix) associated with $\psi_{k}^{(\delta, \alpha)}$.

\section{QUANTUM FLOW}

Consider the symmetries of the state $\psi_{k}^{(\delta, \alpha)}$. By redefining the summation index in Eq. (26), it is straightforward to show that

$$
\psi_{k}^{(\delta+n, \alpha)}(\rho, \varphi)=\psi_{k}^{(\delta, \alpha)}(\rho, \varphi)
$$

from which it follows that integer flux parameters are equivalent to zero. Also,

$$
\psi_{k}^{(-\delta, \alpha)}(\rho, \varphi)=\psi_{k}^{(\delta, \alpha)}(\rho,-\varphi)
$$

implies that for vanishing flux parameter $\psi_{k}^{(0, \alpha)}(r, \theta)$ $\equiv \psi_{k}^{(0, \alpha)}(r,-\theta)$. Thus the quantum flow is symmetric with respect to the $x^{1}$ axis and, consequently, no probability from the upper half-plane passes to the lower half-plane, and vice versa. In other words the current lines associated with the quantum flow must not cross the $x^{1}$ axis; otherwise, due to the symmetry, they would intercept on this axis. When the flux parameter is switched on this symmetry is broken, implying that the flow corresponding to a charged particle is sensitive to the direction (up or down) in which the magnetic flux runs. By studying symmetries (30) and (31), one sees that

$$
0 \leqslant \delta \leqslant \frac{1}{2}
$$

covers all possible behaviors of the flow, and that $\delta=\frac{1}{2}$ also yields a flow symmetric with respect to the $x^{1}$ axis. Expressions (30) and (31) generalize the known symmetries [24,2] of the $\mathrm{AB}$ setup to include the presence of a conical singularity.

Considering Eqs. (26) and (28), the probability density

$$
\begin{aligned}
\psi_{k}^{(\delta, \alpha)} \psi_{k}^{(\delta, \alpha)^{*}}= & \sum_{m, m^{\prime}=-\infty}^{\infty} \exp \left\{i \Theta_{m, m^{\prime}}^{(\delta, \alpha)}(\varphi)\right\} \\
& \times J_{|m-\delta| / \alpha}(k \rho) J_{\left|m^{\prime}-\delta\right| / \alpha}(k \rho)
\end{aligned}
$$

follows, where

$$
\Theta_{m, m^{\prime}}^{(\delta, \alpha)}(\varphi):=\frac{1}{\alpha}\left[\left(|m-\delta|-\left|m^{\prime}-\delta\right|\right) \frac{\pi}{2}-\left(m-m^{\prime}\right) \varphi\right]
$$

satisfying $\Theta_{m, m^{\prime}}^{(\delta, \alpha)}=-\Theta_{m^{\prime}, m}^{(\delta, \alpha)}$. The probability current, when expressed with respect to $\left\{X^{1}, X^{2}\right\}$, has the familiar polar components on the Euclidean plane (18), from which it follows that

$$
\begin{aligned}
\mathcal{J}_{\rho}^{(\delta, \alpha)}(\rho, \varphi)= & \frac{\hbar k}{2 M} \sum_{m, m^{\prime}=-\infty}^{\infty} \sin \left\{\Theta_{m, m^{\prime}}^{(\delta, \alpha)}(\varphi)\right\} J_{|m-\delta| / \alpha}(k \rho) \\
& \times\left[J_{\left|m^{\prime}-\delta\right| / \alpha-1}(k \rho)-J_{\left|m^{\prime}-\delta\right| / \alpha+1}(k \rho)\right]
\end{aligned}
$$

and

$$
\begin{aligned}
\mathcal{J}_{\varphi}^{(\delta, \alpha)}(\rho, \varphi)= & \frac{\hbar}{M \rho_{m, m^{\prime}=-\infty}} \sum^{\infty} \frac{m^{\prime}-\delta}{\alpha} \cos \left\{\Theta_{m, m^{\prime}}^{(\delta, \alpha)}(\varphi)\right\} \\
& \times J_{|m-\delta| / \alpha}(k \rho) J_{\left|m^{\prime}-\delta\right| / \alpha}(k \rho) .
\end{aligned}
$$

In deriving Eq. (35), equality (25) has been used.

In the following the quantum flow will be studied when

$$
k \rho \ll 1 .
$$

This amounts to considering the expansion

$$
J_{\nu}(z)=\left(\frac{z}{2}\right)^{\nu}\left[\frac{1}{\Gamma(1+\nu)}-\frac{1}{\Gamma(2+\nu)}\left(\frac{z}{2}\right)^{2}+O\left(z^{4}\right)\right]
$$

in Eqs. (33), (35), and (36), keeping only the terms with small $m$ and $m^{\prime}$. For simplicity the cone and the flux tube will be considered separately. It should be remarked that Eq. (37) contrasts with the regime on scattering problems for which $k \rho \gg 1$.

\section{A. Conical singularity}

Using Eqs. (34) and (38), it follows from Eq. (33) that the first terms of the probability density around a conical singularity are

$$
\begin{aligned}
\psi_{k}^{(0, \alpha)} \psi_{k}^{(0, \alpha) *}= & 1+\frac{\cos \{\pi / 2 \alpha\} \cos \{\varphi / \alpha\}}{2^{1 / \alpha-2} \Gamma(1+1 / \alpha)}(k \rho)^{1 / \alpha}-\frac{1}{2}(k \rho)^{2} \\
& +\frac{\alpha^{2}}{2^{2 / \alpha-1}}\left[\frac{1+\cos \{2 \varphi / \alpha\}}{\Gamma^{2}(1 / \alpha)}\right. \\
& \left.+\frac{\cos \{\pi / \alpha\} \cos \{2 \varphi / \alpha\}}{\alpha \Gamma(2 / \alpha)}\right](k \rho)^{2 / \alpha}
\end{aligned}
$$

where all terms of the order of $(k \rho)^{\lambda}$, with $\lambda \leqslant 2$ and $\frac{1}{2}<\alpha$ $<\frac{3}{2}$, have been considered. When $\alpha=1$ the corresponding (nonzeroth) powers of $k \rho$ in Eq. (39) cancel out, as they should, since in the absence of the conical singularity (and of magnetic flux) the probing state becomes a plane wave [see Eq. (29)].

In studying the probability density (39), one may be led to conclude that the configuration space of a particle in the state $\psi_{k}^{(0, \alpha)}$ is simply connected, since $\psi_{k}^{(0, \alpha)} \psi_{k}^{(0, \alpha)^{*}}$ does not vanish at the conical singularity (i.e., in the limit $k \rho \rightarrow 0$ ). However, the following considerations may lead to a different interpretation. A way of preventing a particle from getting inside a disc centered at the origin is to impose boundary conditions such that the radial component of the probability current vanishes on the border of the disc (and this may be implemented without requiring that the wave function itself vanishes at the border). Thus no probability leaks into the 
hole, and the current lines surround the disc without crossing it. This is a nonsimply connected configuration space. When the disc degenerates to a point at the origin, this picture does not change. At the origin, which is now the border of the disc, the radial probability current vanishes. It is reasonable to say that the configuration space of this particle is $R^{2}$ $-\{0\}$, although the probability density may be nonvanishing at the origin. If this interpretation is adopted, it follows that before making any statement about the connectivity of the configuration space of a particle on the cone, one should also study the probability current at the singularity.

Before determining the behavior of the probability current at the conical singularity, consider its corresponding quantum potential (A2). The term containing $(k \rho)^{1 / \alpha}$ in Eq. (39) satisfies the Laplace equation and, consequently, does not give any information about the quantum potential [that is the reason why higher order corrections in Eq. (39) were considered]. For $\frac{1}{2}<\alpha<\frac{3}{2}$, it follows from Eqs. (A2) and (39) that the quantum potential near the conical singularity is approximately given by

$$
V_{\alpha}(\rho)=-\frac{(\hbar k)^{2}}{2 M} \frac{(k \rho / 2)^{2 / \alpha-2}}{\Gamma^{2}[1 / \alpha]},
$$

where an unimportant constant has been dropped. The fact that Eq. (40) is not a constant when the conical singularity is present $(\alpha \neq 1)$ constitutes a genuine quantum-mechanical effect (a geometrical Aharonov-Bohm effect). Far away from the conical singularity, for positive $X^{1}$, the state $\psi^{(0, \alpha<1 / 2)}$ behaves approximately as the plane wave (29) [9,7]. Consequently, in terms of $\left\{X^{1}, X^{2}\right\}$, the current lines of the quantum flow are approximately straight lines parallel to the $X^{1}$ axis, running to the left. At this stage, the quantum flow coincides with a flow of classical particles (see Sec. II). As the singularity is approached, the two flows depart from each other. Near the conical singularity they differ radically—by differentiating the quantum potential (40), it follows that the current lines are scattered away from the conical singularity when $\alpha<1$ and bent toward it when $\alpha>1$, whereas the classical particles experience no force (in the $\left\{X^{1}, X^{2}\right\}$ frame). When $\alpha=1$, the quantum potential (40) becomes constant and the classical and quantum flows coincide everywhere, as they must.

After this rather qualitative analysis, the probability current will now be determined near the conical singularity. By proceeding as one did to obtain Eq. (39), from Eqs. (35) and (36) it follows that

$$
\begin{aligned}
\mathcal{J}_{\rho}^{(0, \alpha)}(\rho, \varphi)= & -\frac{\hbar k}{M}\left(\frac{k \rho}{2}\right)^{1 / \alpha-1}\left[\frac{\sin \{\pi / 2 \alpha\} \cos \{\varphi / \alpha\}}{\Gamma(1 / \alpha)}\right. \\
& \left.+\frac{\sin \{\pi / \alpha\} \cos \{2 \varphi / \alpha\}}{2^{1 / \alpha} \Gamma(2 / \alpha)}(k \rho)^{1 / \alpha}\right]
\end{aligned}
$$

$$
\begin{aligned}
\mathcal{J}_{\varphi}^{(0, \alpha)}(\rho, \varphi)= & \frac{\hbar k}{M}\left(\frac{k \rho}{2}\right)^{1 / \alpha-1}\left[\frac{\sin \{\pi / 2 \alpha\} \sin \{\varphi / \alpha\}}{\Gamma(1 / \alpha)}\right. \\
& \left.+\frac{\sin \{\pi / \alpha\} \sin \{2 \varphi / \alpha\}}{2^{1 / \alpha} \Gamma(2 / \alpha)}(k \rho)^{1 / \alpha}\right],
\end{aligned}
$$

where terms $O\left[(k \rho)^{2}\right]$ and $O\left[(k \rho)^{2 / \alpha}\right]$ have been omitted for $\alpha \leqslant 1$ or $\alpha>1$, respectively. When $1 / \alpha$ is even, one sees that expressions (41) and (42) vanish, which is consistent with the fact that the stationary state $\psi_{k}^{(0,1 / 2 n)}$ is a real function [7]. This effect becomes more intuitive by recalling that for even $1 / \alpha$ classical particles are scattered backwards, and then the scattered classical flow cancels the incident one, resulting in a vanishing net classical flow. Clearly when $\alpha=1$, Eqs. (41) and (42) imply that $\mathcal{J}_{\rho}^{(0,1)}(\rho, \varphi)$ $=-(\hbar k / M) \cos \varphi$ and $\mathcal{J}_{\varphi}^{(0,1)}(\rho, \varphi)=(\hbar k / M) \sin \varphi$, which are the polar components of the plane-wave probability current,

$$
\mathcal{J}^{(0,1)}(\rho, \varphi)=-\frac{\hbar k}{M} \mathbf{e}_{1}
$$

$\left[\mathbf{e}_{1}:=(1,0)\right]$, as expected. From Eqs. (41) and (42), one sees that in the limit $\rho \rightarrow 0$ the probability current either vanishes or diverges for $\alpha<1$ and $\alpha>1$, respectively. Note that although $\mathcal{J}_{\rho}^{(0, \alpha>1)}$ diverges as $\rho \rightarrow 0$, the right-hand side of Eq. (17) vanishes, which is not surprising since this was the criterion for choosing the stationary states. The fact that for $\alpha$ $<1$ the quantum flow avoids the conical singularity suggests that the corresponding configuration space is nonsimply connected (and vice versa for $\alpha \geqslant 1$ ), as discussed previously. At this point it should be remarked that according to the authors of Ref. [15], if $\psi \neq 0$ at the conical singularity, the configuration space is simply connected and, consequently, two identical particles on the plane $\left(\alpha=\frac{1}{2}\right)$ in the state $\psi$ "collide." The analysis of the quantum flow above seems to suggest that this may be not the case.

Due to the presence of the wedge in the singular Cartesian coordinates $\left\{X^{1}, X^{2}\right\}$, for some purposes it is more convenient to describe the flow in terms of the embedded Cartesian coordinates $\left\{x^{1}, x^{2}\right\}$. By performing the coordinate transformation $X^{i} \rightarrow x^{i}$ it is straightforward to show that, in the $\left\{x^{1}, x^{2}\right\}$ frame, the probability current is given by $\mathbf{j}=j_{r} \mathbf{e}_{r}$ $+j_{\theta} \mathbf{e}_{\theta}$, with $j_{r}=\alpha \mathcal{J}_{\rho}$ and $j_{\theta}=\mathcal{J}_{\varphi}$. (Note that the wave function and $\mathbf{j}$ satisfy the usual Cartesian form of the continuity equation.) Then, keeping only the leading contribution in Eqs. (41) and (42), one finds

$$
\begin{aligned}
\mathbf{j}^{(0, \alpha)}(r, \theta)= & -\frac{\hbar k}{M}\left(\frac{k r}{2 \alpha}\right)^{1 / \alpha-1} \frac{\sin \{\pi / 2 \alpha\}}{\Gamma(1 / \alpha)} \\
& \times\left[\mathbf{e}_{\mathbf{1}}+(\alpha-1) \cos \theta \mathbf{e}_{r}\right],
\end{aligned}
$$

where the features mentioned above may easily be verified. For example setting $\alpha=1$ in Eq. (44) reproduces Eq. (43).

Figures 5, 6, and 7 show the current lines associated with $\mathbf{j}^{(0, \alpha)}$ for $\alpha<1, \alpha=1$, and $\alpha>1$. They have been obtained by plotting the numerical integration of the equations resulting by equating $d \mathbf{x} / d \lambda$ to the term between brackets in Eq. (44), 


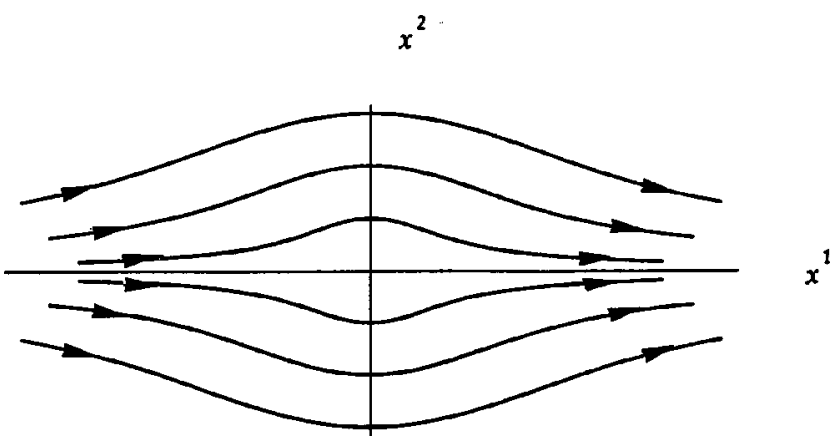

FIG. 5. Quantum flow when $\alpha<1$.

$$
\begin{gathered}
\frac{d x^{1}}{d \lambda}=1+(\alpha-1) \frac{\left(x^{1}\right)^{2}}{\left(x^{1}\right)^{2}+\left(x^{2}\right)^{2}}, \\
\frac{d x^{2}}{d \lambda}=(\alpha-1) \frac{x^{1} x^{2}}{\left(x^{1}\right)^{2}+\left(x^{2}\right)^{2}},
\end{gathered}
$$

where $\lambda$ is an arbitrary parameter.

At $\theta=0, \pm \pi / 2$, and $\pi$, the flow runs parallel to the $x^{1}$ axis, as may be seen from Eq. (44). As the direction of $\mathbf{j}^{(0, \alpha)}$ in Eq. (44) for a given $\alpha$ is determined by $\theta$ only, the current lines are parallel to each other (a feature shared with the classical flow). However, this is true only very close to the singularity, where the subleading contributions in Eqs. (41) and (42) are not relevant. Apart from diverging at the conical singularity for $\alpha>1$, the probability current is smooth everywhere.

Consider more carefully the factor $f(r, \mathcal{D}):=(k r)^{1 / \alpha-1}$ in Eq. (44). For an infinitesimal $\epsilon>0, f(0,0+\epsilon)=0, f(0,0)$ $=1$, and $f(0,0-\epsilon)=\infty$. This discontinuity makes the behavior of the quantum flow on the Euclidean plane change abruptly in the presence of a tiny deficit angle. No such effect exists at the classical level, where the velocity of the particles varies smoothly with the deficit angle. This effect is less surprising when recalling that even a tiny deficit angle $\epsilon$ corresponds to a $\delta$-function curvature which "pierces", the configuration space.

An unsuspected relationship between the winding number of classical orbits and the quantum flow arises when studying the direction of the latter [left or right, with the flow (43) running to the left]. Such direction is determined by the factor $\sin \{\pi / 2 \alpha\}$ in Eq. (44). For $\alpha>\frac{1}{2}$, the quantum flow always runs to the left, and the classical particles are scattered without winding around the conical singularity. For $\alpha=\frac{1}{2}$ (classical backward scattering) the quantum flow stops and reverses its direction for $\alpha<\frac{1}{2}$, with the classical particles winding once around the conical singularity. It continues to run to the right until one decreases $\alpha$ to $\frac{1}{4}$ when another classical backward scattering takes place with another interruption of the flow. By further decreasing $\alpha$, the quantum flow starts to run to the left, while the classical particles wind twice around the conical singularity. Generically, the direction of the quantum flow is controlled by the winding number $w$ defined in Eq. (6) - the quantum flow runs to the left for even $w$ and to the right for odd $w$.

From Fig. 5, one sees that for $\alpha<1$ the quantum flow negotiates the conical singularity in a manner similar to the

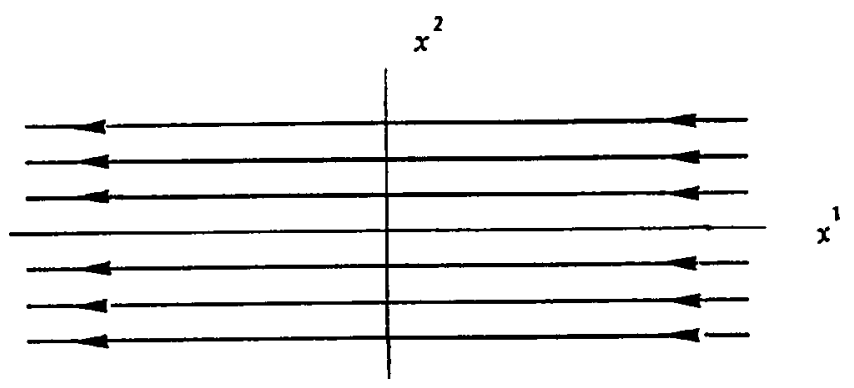

FIG. 6. Quantum flow when $\alpha=1$.

one in which a low velocity fluid negotiates a cylinder. This analogy may be taken as an evidence that the configuration space is not simply connected when $\alpha<1$, as was suggested above. Note that there are no vortices present anywhere since the magnetic flux is switched off [observe Eq. (A5)].

\section{B. Flux tube}

Now consider nonvanishing magnetic flux in the absence of a conical singularity. The analog of Eq. (39) is

$$
\begin{aligned}
\psi_{k}^{(\delta, 1)} \psi_{k}^{(\delta, 1)^{*}=} & \frac{1}{2^{2 \delta} \Gamma^{2}(1+\delta)}(k \rho)^{2 \delta}+\frac{\sin \{\varphi+\delta \pi\}}{\Gamma(1+\delta) \Gamma(2-\delta)} k \rho \\
& -\frac{\sin \varphi}{2^{2 \delta} \Gamma(1+\delta) \Gamma(2+\delta)}(k \rho)^{1+2 \delta} \\
& +\frac{1}{2^{2-2 \delta} \Gamma^{2}(2-\delta)}(k \rho)^{2-2 \delta}
\end{aligned}
$$

where all terms of order $(k \rho)^{\lambda}$ were taken into account, with $\lambda \leqslant 1$, and Eq. (32) was considered. Expression (45) agrees with the one in Ref. [2], where a slightly different method was used. For $\delta=0$, Eq. (45) reduces to unity up to a $(k \rho)^{2}$ term, which would be canceled if higher powers of $k \rho$ in Eq. (45) had been kept. For a nonvanishing flux parameter, the probability density vanishes at the flux tube $(\rho=0)$ and the corresponding configuration space is nonsimply connected. The expression for the quantum potential corresponding to Eq. (45) will not be given here. Instead a study of the probability current itself is carried out in the following.

As in the case of the conical singularity, Eqs. (35) and (36) may be used to obtain $\mathcal{J}_{\rho}(\rho, \varphi)$ and $\mathcal{J}_{\varphi}(\rho, \varphi)$. Expressions which correspond to Eqs. (41) and (42) were previously found in Ref. [2] (see also Ref. [24]). Study will be limited to the case where the flux parameter is very small, viz. $\delta \ll 1$. In so doing, $\mathbf{J}=\mathcal{J}_{\rho} \mathbf{e}_{\rho}+\mathcal{J}_{\varphi} \mathbf{e}_{\varphi}$ reads approximately

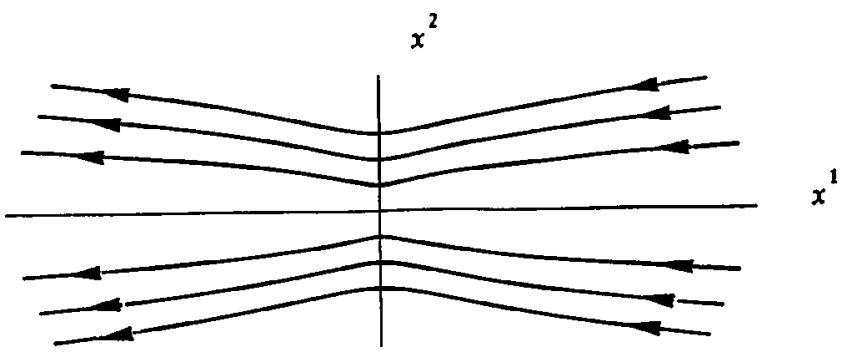

FIG. 7. Quantum flow when $\alpha>1$. 


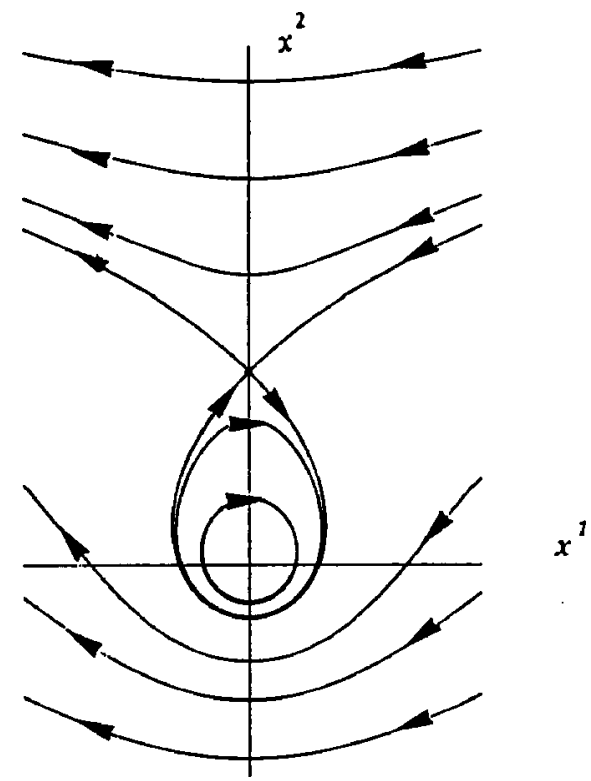

FIG. 8. Quantum flow around a flux tube.

$$
\mathbf{J}^{(\delta, 1)}(\rho, \varphi)=\frac{\hbar k}{M}\left[\mathbf{e}_{\delta}-\frac{\delta}{k \rho} \mathbf{e}_{\varphi}\right]
$$

where $\mathbf{e}_{\delta}$ is the unit vector $\mathbf{e}_{\rho}$ evaluated at $\varphi=\pi(1-\delta / 2)$. When $\delta=0$, Eq. (46) reduces to Eq. (43), as should be. Clearly $\mathbf{J}$ vanishes when $\mathbf{e}_{\varphi}=\mathbf{e}_{\delta}$ and $k \rho=\delta$, so that

$$
(\rho, \varphi)=[\delta / k, \pi(1-\delta) / 2] .
$$

It is also clear from Eq. (46) that the quantum flow (under the action of the corresponding quantum potential) circulates around the origin when it is close to the origin [24]. It turns out that Eq. (47) is a stagnation point, and that there is a vortex around the flux tube, which was expected by observing Eq. (A5). In Fig. 8, the numerical solution of

$$
\begin{gathered}
\frac{d x^{1}}{d \lambda}=-1+(\delta / k) \frac{x^{2}}{\left(x^{1}\right)^{2}+\left(x^{2}\right)^{2}}, \\
\frac{d x^{2}}{d \lambda}=-(\delta / k) \frac{x^{1}}{\left(x^{1}\right)^{2}+\left(x^{2}\right)^{2}}
\end{gathered}
$$

is plotted, showing the main features of the quantum flow near the flux tube. This is in agreement with Ref. [2], where the analytical expression for the current lines was given.

One sees from Eq. (46) that, as with the conical singularity, the $\delta$ function magnetic field at the origin imparts a discontinuous change in the quantum flow of a charged particle. Any tiny amount of magnetic flux changes the topology of the current lines (open lines become loops) near the origin. From Fig. 8 one sees that, as previously mentioned, the presence of the magnetic flux breaks the symmetry with respect to the $X^{1}$ axis. Notice that, as the distance from the origin increases (still for $k \rho \ll 1$ ), the flow gradually aproaches that of a plane wave [Eq. (43)], unlike the effect caused by the conical singularity. An important distinction between the effects caused by the conical singularity and the magnetic flux is that the latter only affects charged particles $(\delta \neq 0)$, whereas the former affects all particles indifferently. This fact may be seen as a manifestation of the equivalence principle-geometrical Aharonov-Bohm effects due to a conical singularity do not depend on any particle attribute.

\section{SUMMARY}

Summarizing, it was shown that the motion of a particle on a flux cone can be regarded as a motion under the action of an angular-momentum-dependent central force. Due to local flatness, quantization was implemented along usual procedures in flat space. By studying the probability fluid corresponding to a particular stationary state ("plane wave", on a flux cone), interesting effects were found. For example, it was shown that the winding number of classical orbits controls the direction of the quantum flow on a cone. Classical flow (which is nearly trivial) and quantum flow were shown to depart from each other near the singularity due to the presence of a nonvanishing quantum force. For the case of a conical singularity, the corresponding quantum potential was determined and analyzed. The issue of connectivity of the configuration space was treated and some interpretations were proposed.

Other boundary conditions at the singularity (those considered in Refs. $[14,15])$ may lead to interesting effects. Another interesting extension of this work would be to consider relativistic particles. The use of this approach in the study of quantum flow in the context of other geometries and topologies would be worthwhile, particularly in geometries with horizons.

\section{ACKNOWLEDGMENTS}

The author is grateful to George Matsas for reviewing the manuscript and for clarifying discussions. The work was supported by FAPESP Grant No. 96/12259-1.

\section{APPENDIX: HYDRODYNAMICAL APPROACH TO QUANTUM MECHANICS}

Analogies between quantum mechanics and fluid dynamics do not stop at a continuity equation which expresses local conservation of probability. The Schrödinger equation can be rephrased as a set of hydrodynamical equations (see Ref. [2], and references therein). In order to derive them, consider a particle with charge $e$ moving in the Euclidean space (the generalization to arbitrary backgrounds is straightforward) under the action of an electromagnetic field (E,B) with corresponding potentials $(\boldsymbol{\phi}, \mathbf{A})$. The Hamiltonian operator is then given by

$$
H=\frac{1}{2 M}\left(-i \hbar \nabla-\frac{e}{c} \mathbf{A}\right)^{2}+e \phi .
$$

Now one rewrites the solution $\psi$ for the corresponding Schrödinger equation as

$$
\psi(t, \mathbf{r})=\varrho(t, \mathbf{r}) e^{i \chi(t, \mathbf{r})} .
$$

The real part of the Schrödinger equation reads 


$$
\hbar \frac{\partial \chi}{\partial t}+\frac{\hbar^{2}}{2 M}\left[\boldsymbol{\nabla} \chi-\frac{e}{c \hbar} \mathbf{A}\right]^{2}+e \phi+V=0
$$

where

$$
\begin{aligned}
V(t, \mathbf{r}): & =-\frac{\hbar^{2}}{2 M} \frac{\nabla^{2} \varrho}{\varrho} \\
& \equiv-\frac{\hbar^{2}}{2 M} \frac{\nabla^{2}\left(\psi \psi^{*}\right)^{1 / 2}}{\left(\psi \psi^{*}\right)^{1 / 2}} .
\end{aligned}
$$

The imaginary part reads

$$
\frac{\partial \varrho^{2}}{\partial t}+\nabla \cdot\left(\varrho^{2} \mathbf{v}\right)=0
$$

with $\mathbf{v}:=[\hbar \nabla \chi-e \mathbf{A} / c] / M$, leading to the constraint

$$
\boldsymbol{\nabla} \times \mathbf{v}=-\frac{e}{M c} \mathbf{B}
$$

Now one sees that $\varrho^{2} \mathbf{v}$ is the probability current, and therefore Eq. (A3) is just the continuity equation. Note that by integrating Eq. (A4) along a closed loop, it follows that

$$
\oint \mathbf{v} \cdot d \mathbf{l}=-\frac{e \Phi}{M c}
$$

where $\Phi$ is the magnetic flux enclosed by the loop.

The last step is to apply $\boldsymbol{\nabla}$ in Eq. (A1), after which one is left with a set of equations of motion for a fluid of density $\varrho^{2}$ and velocity $\mathbf{v}$,

$$
\begin{aligned}
M \frac{d}{d t} \mathbf{v}(t, \mathbf{r}) & \equiv M\left[\frac{\partial \mathbf{v}}{\partial t}+(\mathbf{v} \cdot \boldsymbol{\nabla}) \mathbf{v}\right] \\
& =e \mathbf{E}+\frac{e}{c} \mathbf{v} \times \mathbf{B}-\nabla V .
\end{aligned}
$$

Thus the wavelike Schrödinger equation [where the function to be determined is $\psi$ for a given configuration of potentials $(\phi, \mathbf{A})]$ has been replaced by the hydrodynamical equations (A3) and (A6) with the constraint (A4) [where the functions to be determined are $(\varrho, \mathbf{v})$ for a given configuration of electromagnetic field $(\mathbf{E}, \mathbf{B})]$. These equations govern the behavior of the quantum flow.

Whereas the Schrödinger equation is more appropriate to study wavelike features of quantum mechanics, the hydrodynamical equations are more appropriate to study particlelike features. To see this, assume that the quantum force $-\nabla V$ in Eq. (A6) is negligible when compared with the Lorentz force (and other forces that one might have considered). In this "classical limit," Eq. (A6) reduces to the equations of motion for a flow of noninteracting classical particles. Note that Eq. (A1) is the corresponding Hamilton-Jacobi equation, where the action is identified with $\hbar \chi$. It is clear that the quantum potential $V$ represents the departure from the classical motion. In regions where the quantum potential is relevant, the classical and quantum flows may differ considerably from each other. For example, in a region of vanishing field strengths the motion of the classical flow is trivial, since the Lorentz force vanishes there. The motion of the quantum flow, on the other hand, may be quite elaborate due to the presence of the quantum force $-\nabla V$ in Eq. (A6). A nonvanishing quantum force is the essence of Aharonov-Bohmlike effects.
[1] Y. Aharanov and D. Bohm, Phys. Rev. 115, 485 (1959).

[2] S. Olariu and I. I. Popescu, Rev. Mod. Phys. 57, 339 (1985).

[3] L. Marder, Proc. R. Soc. London, Ser. A 252, 45 (1959).

[4] A. Staruszkiewicz, Acta Phys. Pol. 24, 734 (1963).

[5] J. S. Dowker, Nuovo Cimento B 52, 129 (1967).

[6] G. 't Hooft, Commun. Math. Phys. 117, 685 (1988).

[7] S. Deser and R. Jackiw, Commun. Math. Phys. 118, 495 (1988)

[8] P. S. Gerbert and R. Jackiw, Commun. Math. Phys. 124, 229 (1989); P. S. Gerbert, Nucl. Phys. B 346, 440 (1990).

[9] D. Lancaster, Ph.D. thesis, Stanford University, (1984); Phys. Rev. D 42, 2678 (1990).

[10] J. S. Dowker, J. Phys. A 10, 115 (1977); Phys. Rev. D 18, 1856 (1978).

[11] A. G. Smith, in The Formation and Evolution of Cosmic Strings, edited by G. W. Gibbons, S. W. Hawking, and T. Vachaspati (Cambridge University Press, Cambridge, 1990); T. M. Helliwell and D. A. Konkowski, Phys. Rev. D 34, 1918 (1986); B. Linet, ibid. 35, 536 (1987); V. P. Frolov and E. M. Serebriany, ibid. 35, 3779 (1987); J. S. Dowker, ibid. 36, 3095 (1987); 36, 3742 (1987).
[12] S. Zerbini, G. Cognola, and L. Vanzo, Phys. Rev. D 54, 2699 (1996).

[13] S. Deser, R. Jackiw, and G. 't Hooft, Ann. Phys. (N.Y.) 152, 220 (1984).

[14] B. S. Kay and U. M. Studer, Commun. Math. Phys. 139, 103 (1991).

[15] M. Bourdeau and R. D. Sorkin, Phys. Rev. D 45, 687 (1992).

[16] D. D. Sokolov and A. A. Starobinskii, Dok. Akad. Nauk 234, 1043 (1977) [Sov. Phys. Dokl. 22, 312 (1977)].

[17] E. S. Moreira, Jr., Ph.D. thesis, University of London, 1997.

[18] G. Clément, Int. J. Theor. Phys. 24, 267 (1985).

[19] R. Jackiw and A. N. Redlich, Phys. Rev. Lett. 50, 555 (1983).

[20] B. Felsager, Geometry, Particles and Fields (Odense University Press, Odense, 1981).

[21] V. B. Bezerra, J. Math. Phys. 30, 2895 (1989).

[22] P. A. M. Dirac, Proc. R. Soc. London, Ser. A 133, 60 (1931).

[23] E. S. Moreira, Jr., Nucl. Phys. B 451, 365 (1995).

[24] M. V. Berry, R. G. Chambers, M. G. Large, C. Upstill, and J. C. Walmsley, Eur. J. Phys. 1, 154 (1980). 